
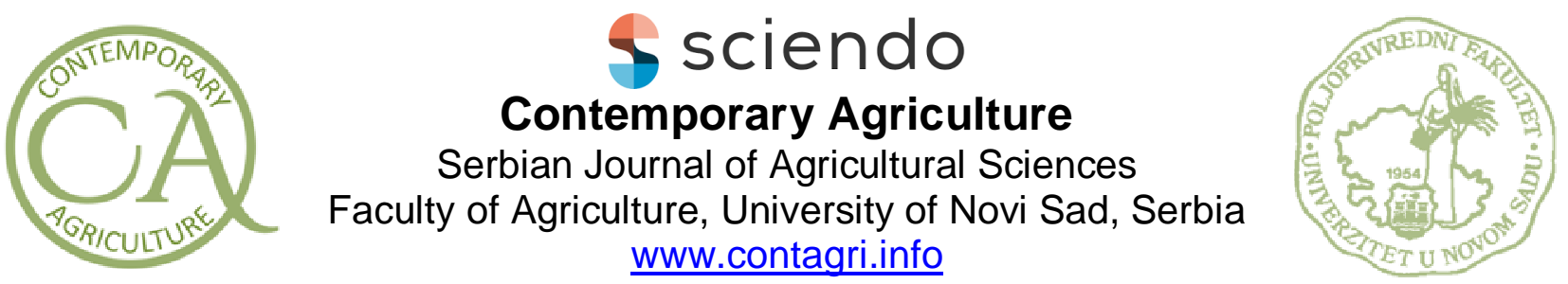

Original scientific paper

UDC: 619:614.253

DOI: $10.2478 /$ contagri-2021-0004

\title{
WORK PERFORMANCE OF THE ETHICS COMMITTEE FOR THE WELFARE OF ANIMALS USED IN ANIMAL TESTING OF THE UNIVERSITY OF NOVI SAD FROM 2016 TO 2018
}

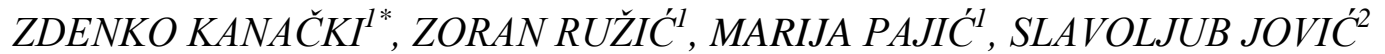 \\ ${ }^{1}$ Department of Veterinary Medicine, Faculty of Agriculture, University of Novi Sad, Trg Dositeja Obradovića 8, \\ 21000 Novi Sad, Serbia \\ ${ }^{2}$ Faculty of Veterinary Medicine, University of Belgrade, Bulevar Oslobođenja 18, 11000 Belgrade, Serbia \\ "Corresponding author: zdenkokanacki@gmail.com
}

\begin{abstract}
SUMMARY
An animal experiment can be defined as any procedure in which a live animal is used with the aim of testing a scientific assumption, gathering information, obtaining or testing substances or observing the effect of a particular measure on the animal, as well as using an animal in behavioral experiments. The Ethics Committee (EC) determines how animal testing shall be conducted and, in accordance with the Law on Animal Welfare, provides expert supervision of animal testing, organizes training courses for persons conducting testing, provides expert opinions to the minister regarding ethical and scientific justification of animal testing, and submits annual reports to the minister. The data used in this paper were derived in the course of work of the EC in the period between 2016 and 2018. The meetings of the EC were held on a regular basis and in accordance with the Rules of Procedure. Positive Opinions were issued for most of the submitted Applications (87.5\%), while a smaller number was returned $(6.25 \%)$ or directed to the Council for further consideration (6.25\%). In several cases, the EC also issued Opinions that certain experiments are beyond its scope of authority and thus beyond its formal legal capacity. Based on the Application of a candidate, the EC also approved changes in previously issued Opinion to ensure consistency in the documentation. The Committee regularly submitted reports and there were no objections to its work. In accordance with the abovementioned, the performance of the EC can be considered efficient and successful, but it can be further significantly improved by more active effort of the individual members of the EC within their home institutions to educate candidates and raise awareness of the importance of the work performed by the EC.
\end{abstract}

Key words: Law on Animal Welfare, Ethics Committee, animal testing, laboratory animals

\section{INTRODUCTION}

An animal experiment can be defined as any procedure in which a live animal is used with the aim of testing a scientific assumption, gathering information, obtaining or testing substances or observing the effect of a particular measure on the animal, as well as using an animal in behavioral experiments (as defined by the Swiss Animal Protection Act). The Law on Animal Welfare was adopted by the Republic of Serbia in 2009, establishing a system in the field of animal testing in accordance with the system already implemented in the EU. Following the Law, appropriate by-laws were also passed, closely defining specific issues. This system integrates all persons involved in the experiment, from the relevant ministry to the researchers and support personnel. It puts in place a system for the control of the use of animals in animal testing.

The Law, especially Article 5, defines animals used in animal testing as all live vertebrates and invertebrates in all stages of development. The other articles of the Law pertaining to animal testing, Ethics Council, and Ethics Committees (EC) are Articles 33-52. Animal testing is approved by the relevant minister, based on an expert opinion 
provided by the local EC. In accordance with the Law, animal testing may be performed only by subjects entered into the register of the relevant ministry - Register for Animal Testing (The Law on Animal Welfare, 2009).

With the aim of discussing issues pertaining to the area and providing expert opinions, as well as executing project tasks related to animal welfare, a special work group was formed as the Ethics Council for the Welfare of Animals Used in Animal Testing. In addition, any scientific research organization and all other legal entities conducting animal testing must establish an ethics committee for the welfare of animals used in animal testing within the organization or in collaboration with other scientific research organizations, i.e. legal entities conducting animal testing. The Ethics Committee for the Welfare of Animals Used in Animal Testing of the University of Novi Sad was established for this purpose. The EC and the Ethics Council represent a starting point for ensuring the protection of animals used in animal testing, which is further enforced by other government institutions as well (inspection agencies, the police, the prosecutor's office, and the justice system) and Laws (Veterinary Law, 2005; 2010; 2012).

The Law stipulates that the EC shall consist of veterinary surgeons, veterinarians with experience in breeding animals used in animal testing, experts with experience in implementing statistics in research, representatives of associations, i.e. organizations aimed at protecting animal welfare, as well as researchers in associated scientific fields. In order to include also the opinion of persons not directly involved in scientific research, at least one third of the members of the EC shall consist of persons not employed in a scientific research organization or a different legal entity conducting animal testing (The Law on Animal Welfare, 2009).

The EC determines the manner in which animal testing shall be conducted and, in accordance with the Law on Animal Welfare (2009), provides expert supervision for animal testing, organizes training courses for persons conducting animal testing, provides expert opinions to the minister regarding ethical and scientific justification of animal testing, and submits regular annual reports to the minister. The EC is obliged to terminate an experiment involving animals without delay if the experiment is conducted contrary to the provisions of the Law on Animal Welfare (2009), as well as to inform the Ministry of such misconduct.

The utilitarian approach is still used as a basic principle in the course of work and decision making process of the EC for the welfare of animals used in animal testing. This approach also forms a basis for the creation of a principle called "the Three Rs (3Rs)" which provides the foundation for ensuring the welfare of animals used in animal testing. This principle was introduced by William \& Burch (1959) in their book.

The Rulebook for Work with Experimental Animals of the University of Novi Sad (2011) determines the protected animal species, the experimental procedures (ethical and unethical), the ethical principles for the experiments involving animal subjects, the qualifications of researchers for such work, the membership and the procedure for establishing the EC for the Welfare of Animals used in Animal Testing of the University of Novi Sad, its scope of work, tasks and work rules, the procedure for obtaining the opinion of the EC regarding animal testing, the procedure to be followed in case of noncompliance with the work rules of the EC and the decisions made in accordance with the Rulebook.

The protected animal species include all vertebrates, except humans, that experience pain, suffering, fear and stress, in all stages of development. Ethical experimental procedures refer to the procedures involving the manipulation of animals used for animal testing for the purposes of scientific research and biomedical purposes in order to generate new knowledge in the field of biomedicine, thus contributing to the general development of science. Interventions on animals which cause damage to the physical, mental or genetic state of the animals can only be performed by a veterinarian or an authorized scientist. Unethical experimental procedures refer to all manipulations of animals performed in the course of an experiment which may cause pain, suffering, permanent damage or death, with no purpose of generating new knowledge in the field of biomedicine, i.e. contributing to the development of science. Furthermore, unethical experimental procedures include demonstrations of previously obtained knowledge by performing experiments on animals, except in the cases when the involvement of animals is crucial for achieving education goals. Instead, the use of computer simulations and other generally accepted didactic methods is recommended. Within institutions of higher education, in the course of undergraduate studies, practicing on animals is allowed as long as it does not cause pain, suffering, fear, stress, injury or death of the animal, so as long as life and welfare, i.e. physical, mental, or genetic state of the animal is not jeopardized (Liu et al., 2008; Liu, 2014; Qin \& Wei, 2015).

When discussing applications, the EC evaluates the justifications in accordance with the following ethics principles (Enqi \& Jianglin, 2018; Kanački \& Pajić, 2019):

- Replacement principle: whenever possible, instead of in vivo experiments involving a protected species, alternative experimental in vitro models (e.g. cell cultures, isollated organs, microorganisms) and computer simulations shall be used. 
- Reduction principle: the smallest possible number of animals shall be used in the experiment in accordance with the statistical calculations.

- Refinement principle: experimental procedures shall be improved up to a point at which it shall be possible to completely avoid or maximally reduce the unpleasant physical and emotional experience of the animal used in the experiment.

In addition, the EC evaluates other conditions related to the researchers, the origin of the animals used in animal testing and the processing of the obtained results. Researchers should be trained to work on experiments involving animals, and the required qualifications shall depend on a specific experimental protocol (Stevanović, 2002; 2004). Researchers are required to submit proof of their qualifications to perform experiments involving animals (certificate of attendance proving they attended a training course in the country or abroad). Experienced researchers can prove their competence by submitting papers published in an international journal. Animals used for animal testing must be bred in professional breeding facilities, and obtained, without exception, from professional breeding facilities, i.e. the facilities supplying legal entities and natural persons - the companies listed in the Register for Animal Testing. Experiments may only involve animals bred exclusively for this purpose. Appropriate statistical methods must be used when evaluating results (The Rulebook for Work with Experimental Animals, 2011).

\section{MATERIAL AND METHODS}

The data used in this paper are the publicly available data derived in the course of work of the EC in the period between 2016 and 2018. The duration of the term of office for the members of the EC is 3 years, which is the reason why this particular period was chosen for the analysis. Regular meetings of the EC take place four times a year, during the third week of March, June, September and December, while the Applications for the approval to conduct animal testing are submitted until the end of the preceding month, i.e. until the end of February for the March meeting, until the end of May for the June meeting, until the end of August for the September meeting and until the end of November for the December meeting. If necessary, meetings may be called more frequently. The call for a meeting is made at least seven days before the day when the meeting is due to take place. The call is accompanied by the proposal of the agenda, minutes from the previous meeting of the EC, and the required accompanying material for each item of the agenda. In case of absence of a quorum, the meeting of the EC shall be postponed. Minutes are kept at every meeting, and they are approved at the following meeting of the EC. The minutes and all accompanying material (Applications, Opinions etc.) of an EC meeting are archived in a separate folder and kept by the Chairperson of the EC as archival material for at least five years (Rules of Procedure of the Ethics Committee for the Protection of Experimental Animal Welfare, 2013).

In addition, the EC determines the procedure for conducting animal testing and provides expert supervision over experiments in accordance with the Law on Animal Welfare (2009); based on available information, it informs the persons conducting the animal testing about courses and seminars regarding animal welfare; it provides the minister with expert opinions on the issues of ethical and scientific justification for conducting an experiment; it submits regular annual reports to the relevant ministry.

Having insight into the publicly available data, the following indicators of the manner and efficiency of the Committee's performance were observed:

- Regular organization of meetings,

- Number and type of Applications considered,

- Number of positive and negative Opinions issued,

- Number of appeals directed at the Ethics Council for consideration of individual Applications,

- Other concurrent tasks of the EC.

The obtained results are shown in the Table 1 . and statistically processed.

\section{RESULTS AND DISCUSSION}

Table 1. Results of the work performed by the EC of the University of Novi Sad from 2016 to 2018

\begin{tabular}{cccccccc}
\hline Year & $\begin{array}{c}\text { No. of } \\
\text { meetings } \\
\text { held }\end{array}$ & $\begin{array}{c}\text { Total no. of } \\
\text { Applications } \\
\text { considered }\end{array}$ & $\begin{array}{c}\text { Positive } \\
\text { Opinion }\end{array}$ & $\begin{array}{c}\text { Negative } \\
\text { Opinion } \\
\text { (returned) }\end{array}$ & $\begin{array}{c}\text { Directed at } \\
\text { the Ethics } \\
\text { Council }\end{array}$ & $\begin{array}{c}\text { Not under the } \\
\text { authority of the } \\
\text { Committee }\end{array}$ & Other \\
\hline 2016 & 4 & 16 & 11 & 1 & 1 & 2 & 1 \\
\hline 2017 & 4 & 8 & 4 & 1 & 0 & 3 & 0 \\
\hline 2018 & 4 & 18 & 13 & 0 & 1 & 4 & 0 \\
\hline
\end{tabular}


In accordance with the Rulebook for the Work Performed by the EC (2011), regular meetings shall be held four times a year, but can be called more often if necessary. Due to the relatively small number of Applications submitted per meeting (3.5 on average), it can be concluded that holding regular meetings provides for normal and uninterrupted work and timely issue of Opinions to the candidates, so the additional meetings were not necessary in this period. In addition to considering Applications, in the course of regular meetings, decisions on other issues regarding the work of the $\mathrm{EC}$ were made, e.g. establishing the managing board, initiating the nomination of new members to replace the members who are reaching the end of their terms, etc.

The EC received a total number of 42 Applications for consideration in this three-year period, i.e. 14 Applications per year on average. The largest number of Applications involved a request for Opinion on the ethical justification of an experiment involving animals - the total of 32 Applications, while 9 Applications referred to a request for Opinion on an experiment involving a subject which was not under the authority of the EC. Only one Application involved a change in the name of a project which had already received a positive Opinion of the EC. During this period of three years, EC did not organize training courses for persons conducting animal testing, because the Ministry announced no calls for training.

In cases where an Application was submitted in order to obtain an Opinion on the ethical justification of animal testing, the applicants were obliged to submit an Official Appeal, a completed Form, and 5 scientific papers discussing a similar issue. The candidates who anticipated that the subject of their research would not be considered to be under the authority of the EC submitted an Official Appeal and the experiment protocol as well. In case incomplete documentation was submitted, the Chairperson of the Committee would notify the applicants of this issue in a timely manner, ensuring that Applications with complete documentation were discussed at the meetings.

Out of the total number of 42 Applications submitted to obtain the Opinion on the justification of the use of animals in animal testing, positive Opinion was issued for 28 Applications (87.5\%), including the Applications which received a positive Opinion with certain corrections. In these cases, the candidates were obliged to submit the corrected Applications to the EC prior to receiving the Opinion, which was done without exception. This kind of procedure was applied to the Applications which contained technical issues or certain imprecision. In this manner, the EC increased efficiency and the candidates' time was not lost waiting for the following meeting of the EC. Based on the obtained positive Opinion of the EC, the candidates were able to receive a Decision from the relevant Ministry allowing them to conduct the experiment. In addition, the candidates were obliged to inform the EC of the beginning of the experiment no later than one week in advance, in order to provide for the supervision of the EC (The Rulebook for Work with Experimental Animals, 2011; Rules of Procedure of the Ethics Committee for the Protection of Experimental Animal Welfare, 2013).

In cases of Applications containing major inconsistencies or inaccuracies, i.e. experiment protocol was considered unacceptable in the submitted form, the EC made the decision to return the Applications to the candidates to be reworked and corrected, and afterwards included the Applications in the agenda of one of the following meetings. There were a relatively small number of such Applications in this period, namely only 2, i.e. $6.25 \%$.

In addition to the Applications which received a positive Opinion and the returned ones, two Applications received the Opinion of the EC that the experiments in question included a higher category of invasiveness than the one stated in the Form. Since the EC concluded that these cases involved E category of invasiveness, which can only be discussed by the Ethics Council, the EC submitted an appeal to the Council to take these Applications into consideration. Based on the received feedback, the Ethics Council has given a positive Opinion for one experiment, while the other one is still under consideration.

Out of the total number of 42 Applications in this period, 9 (21.4\%) involved requests for Opinions for experiments conducted on biological material which is not under the authority of the EC. There are several objective and subjective reasons why candidates chose to address the EC with these Applications. Firstly, several candidates were not aware of the scope of authority of the EC, thus considering it appropriate for the EC to evaluate whether it was necessary to obtain a Decision to conduct such experiments. In this case, an efficient method would be to consult a member of the EC representing the institution within which the candidate is conducting the experiment. This would reduce the scope of work performed by the EC and save time. Secondly, certain institutions of higher education required that doctoral candidates obtain a positive Opinion of the $\mathrm{EC}$ to be able to submit their thesis. This is also not justified, since the Opinion of the EC is not a final act, but rather a basis for obtaining the final Decision from the relevant ministry. Thirdly, certain journals required a positive Opinion of the EC to be submitted along with a paper. The reason for this can be found in different procedures in different countries, based on the fact that most of the cases involved foreign journals. In all of these cases, the EC decided to cooperate with the candidates and provide appropriate Opinions. 
The biological material which was used in these experiments, but was not considered to be under the authority of the $\mathrm{EC}$, included the material derived in the course of a routine veterinary or technological procedure, material obtained from previous experiments (which had been approved), as well as other material excluded from the definition of the Committee's scope of authority.

Lastly, only one Application (2.4\%) referred to the title change of an already approved experiment. This case involved a title correction and the necessity to adjust all documentation to this change to ensure consistency. This is the reason why it is necessary to provide correct data in the Application with regards to the researcher, institution, title of the paper, as well as whether it is a scientific research, a doctoral thesis, etc.

Finally, the work of EC complied with the set period of 45 days from the day of submission to the day of the issue of the Opinion in all cases. The EC submitted regular annual reports on its work to the relevant Ministry, as well as to the Senate of the University of Novi Sad, which approved them. In addition, during this period there were no objections to the work of the EC.

\section{CONCLUSION}

Based on the obtained data, the following conclusions may be reached regarding the performance of the EC for the Welfare of Animals Used in Animal Testing of the University of Novi Sad from 2016 to 2018:

- The meetings of the EC were held on a regular basis and in accordance with the Rules of Procedure.

- Positive Opinions were issued for most of the submitted Applications (87.5\%), while a smaller number of Applications was returned (6.25\%) or directed to the Council for further consideration $(6.25 \%)$.

- In several cases, the EC also issued Opinions that certain experiments are beyond its scope of authority and thus beyond its formal legal capacity.

- Based on the Application of a candidate, the EC also approved changes in previously issued Opinion in order to ensure consistency in the documentation.

- The EC regularly submitted reports and there were no objections to its work.

In accordance with the abovementioned, the performance of the EC can be considered efficient and successful, but it can be further significantly improved by more active effort of the individual members of the EC within their home institutions with the aim to educate candidates and raise awareness of the importance of the work performed by the Committee.

\section{REFERENCES}

Enqi L. \& Jianglin F. (2018): Fundamentals of laboratory animal science. CRC Press Taylor \& Francis Group, Boca Raton, FL, USA.

Kanački Z. \& Pajić M. (2019): Veterinarska etika. Poljoprivredni fakultet u Novom Sadu.

Liu E. (2014): Animal models of human diseases. Second Edition. People's Health Publishing House, Beijing, China.

Liu E., Yin H., Gu W. (2008): Medical Laboratory Animals. Science Press, Beijing, China.

Qin C. \& Wei H. (2015): Laboratory Animal Science. Second Edition. People's Medical Publishing House, Beijing, China.

Russell W.M.S., \& Burch R. (1959): The Principles of Humane Experimental Technique. London, UK: Methuen.

The Rulebook for Work with Experimental Animals, University of Novi Sad, 2011.

Rules of Procedure of the Ethics Committee for the Protection of Experimental Animal Welfare, University of Novi Sad, 2013.

Stevanović Đ. (2002): Osnovi nauke o laboratorijskim životinjama. Dr Stevanović, Beograd.

Stevanović Đ. (2004): Metode i tehnike eksperimentalnog rada sa laboratorijskim životinjama. Dr Stevanović, Beograd.

The Law on Animal Welfare, Official Gazette of the Republic of Serbia No.41/2009

Veterinary Law, Official Gazette of the Republic of Serbia. 91/2005, 30/2010 and 93/2012

Submitted: 23.09.2020.

Accepted: 15.12.2020. 